\title{
Reliable behaviour simulation of product interface in mixed reality
}

\author{
Fabio Bruno $\cdot$ Agostino Angilica $\cdot$ Francesco Cosco • \\ Maurizio Muzzupappa
}

Received: 10 November 2010/ Accepted: 12 October 2012

(C) Springer-Verlag London 2012

\begin{abstract}
The validation of a product interface is often a critical issue in the design process. Virtual reality and mixed reality (MR) are able to enhance the interactive simulation of the product human-machine interface (HMI), as these technologies allow engineers to directly involve end users in the usability assessment. This paper describes a MR environment specifically addressed to the usability evaluation of a product interface, which allows the simulation of the HMI behaviour using the same models and the same software employed by engineers during the design phase. Our approach is based on the run-time connection between the visualisation software and the simulators used for product design and analysis. In particular, we use Matlab/Simulink to model and simulate the product behaviour, and Virtools to create the interactive MR environment in which the end user can test the product. Thanks to this architecture, any modification done on the behaviour models is immediately testable in MR.
\end{abstract}

Keywords Product design evaluation .

Interactive virtual prototype $\cdot$ Functional behaviour simulation $\cdot$ Finite state machine $\cdot$ Mixed reality

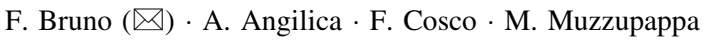
Department of Mechanical Engineering, University of Calabria, Rende, Italy

e-mail: f.bruno@unical.it

URL: www.unical.it
A. Angilica
e-mail: agostino.angilica@unical.it
F. Cosco
e-mail: francesco.cosco@unical.it
M. Muzzupappa
e-mail: muzzupappa@unical.it

\section{Introduction}

Virtual reality (VR) technologies are widely employed for the aesthetical validation of industrial products. VR applications, in fact, provide a high quality, immersive visual representation of virtual prototypes, and designers can easily evaluate aesthetical qualities and/or discover any styling defect [1]. VR has also demonstrated to be a valid tool for usability analyses, because it is able to support and facilitate the participatory design of industrial products. VR allows designers to involve the end users of a product at the early design stages, without the need of a physical mock-up and with the advantage of being able to assess several design options [1-3]. The use of mixed reality (MR) in the product development process is emerging as a promising solution that combines the advantages of virtual prototyping (to quickly evaluate various design alternatives) and physical prototyping (to involve the sense of touch). A mixed prototype is usually based on a physical mock-up on which the visual appearance of the product is superimposed thanks to the augmented reality technologies [4-7].

Unfortunately, visualisation software for VR/MR is mainly addressed to the aesthetical validation of the product. The functional simulation is limited to some basic behaviours, such as movements triggered by an event (e.g. a door will open if the end user clicks on a handle). As a consequence, the obtained behaviour is an animation and not a simulation, so it cannot be considered as a robust validation tool. Furthermore, in VR/MR the behaviour of the product is described by a model implemented inside the VR software [2-7]. This means that each change in the digital mock-up has to be manually reported in the code of the VR application, thus requiring a big effort by the VR operators. In previous works, we approached the problem of simulating the behaviour of mechatronic products in VR 
$[8,9]$ through a software tool that links a multi-body solver with a VR environment.

This paper explores this concept to deal with the problems related to the evaluation of product usability in VR/ MR. The most notable unmet need is the possibility to have a reliable simulation of the HMI behaviour inside a VR/ MR environment. For this need to be met the simulation has to be performed by a solver with a level of precision acceptable for industries and the behaviour models have to be exactly the same as the ones engineers use to describe the product.

Although our approach can be adopted both in VR and MR environments, we have chosen a MR environment that is able to overcome some of the limits of VR, such as the need of dedicated hardware and the lack of tactile feedback. VR applications, in fact, still require complex and expensive devices like tracking systems, data-gloves, or haptic devices needed to give the user the possibility to perceive the tactile feedback while he/she is interacting with a product. The MR set-up that we have adopted employs a video see-through head mounted display (HMD) for augmented visualisation. The occlusion management was solved using a skin-detection algorithm and the recognition of the user gestures was done thanks to a custommade interactive thimble.

The next section provides a review of the state of the art. The review is focused on the main issues related to the product behaviour simulation in MR environments. We also briefly describe some of the most interesting works on mixed prototyping. Sections 3 and 4 present, respectively, the MR environment and the solution proposed for the behaviour simulation. Section 5 reports a test case through which the creation of an interactive digital prototype is described in detail. The last section reports the results of the test aimed at evaluating the acceptability among the end users of the proposed environment.

\section{Related works}

The virtual prototyping approach allows designers and engineers to evaluate their design choices simulating the behaviour of the product since the early stages of the development process. VR and MR technologies support this activity by making the interaction with the digital prototype easier and more natural.

The first mixed prototyping applications allowed to augment a physical mock-up with the visualisation of the surface texture using a see-through HMD [10].

Later on, several mixed prototyping practices were experimented for rapid design review of new products and, in particular, for information appliances. In [5], authors use MR for evaluating some ergonomics aspects of interactive devices. Since only the physical ergonomics is relevant for the purpose of this work, authors do not need to simulate the behaviour of the products. Verlinden and Horvath [11-13] describe a multiple case study addressed to assess the benefits of the mixed prototyping approach. They have also proposed a framework for testing and validating "interactive augmented prototyping", which they define as "the combination of physical and virtual artefact models through augmented reality and rapid prototyping". They adopt the projection-based AR that uses projectors to cast computer imagery directly on physical objects, but it is apparent that this technique has some limitations when the user occludes the projection with his/her hands. Moreover, the proposed approach does not take into account the issues that usually arise when one has to simulate the product behaviour.

In [4], the authors present a MR environment that integrates ARToolkit with an electromagnetic tracker. They focus the attention only on hand-held products, and they assume that the user has to press the buttons of the product only using his/her thumb. Authors do not discuss the issues related to the behaviour simulation.

In [6], a study about the use of VR in product design evaluation is presented. The authors focus on the analysis of the human-machine interfaces (HMI) through a state transition methodology that is addressed to define a behaviour model for the functional simulation. The authors put in evidence that one of the limits of their approach is that "it is very meaningful and challenging to devise an efficient way to reduce the time and effort required to generate the HMI behaviour model of the product by reusing the information built in the embedded software". In a recent progress of [6], the same authors propose an extension of their approach adopting tangible interfaces and AR visualisation [7]. They use rapid prototyping to create a physical mock-up, as also suggested in [4], and they paste the markers needed for ARToolkit tracking on the physical model. The interaction with this augmented tangible prototype is done through a paper pen on which some ARToolkit markers are placed. The authors still adopt the same methodology proposed in their previous work to simulate the product behaviour.

Kanai and Higuchi [14] present an environment that supports both design and testing of product interfaces with 3D digital prototypes. The modelling of the product behaviour is done through an add-on for Microsoft Visio based on the Statechart [15]. The behaviour model can be linked to the 3D model to create an interactive model of the product. This model can be used in some testing activities with end users for usability assessment. The environment also supports the testing phase and the subsequent analysis of the results. From a methodological point of view, this work provides an organic view to the problem of usability assessment, supporting all the phases from design to result 
analysis. On the other hand, it requires the use of a custommade tool for behaviour modelling, while commercial solutions for the design and simulation of interface behaviour already exist (e.g. Simulink). In [16], the authors extend their idea including the support for an XML based language based on UsiXML. This makes the modelling and the testing of the interface behaviour more effective and reliable.

The previously mentioned papers prove that there is a lack of software tools able to support designers in the development of interactive and functional virtual prototypes. Engineers, in fact, use specialised simulation software to design industrial products. At the moment, it is not possible to directly evaluate in MR the models developed in these simulation packages, therefore, it is necessary to work out a specific solution for the several problems that occur during the integration between MR and the other applications of the product development process, including the simulation.

In this paper, we present a research work aimed to the development of a MR environment for the functional simulation of industrial products, mainly addressed to the usability evaluation. The first step in this research was the development of a software library for the run-time connection between the VR software and the simulators used for product design and analysis [17]. In particular, we have chosen Matlab/Simulink to model and simulate the HMI behaviour, and Virtools to create the interactive virtual environment. These two environments are linked through a socket connection to exchange data at run-time, so that the Simulink models may control the behaviour of the digital prototype animated in Virtools. This approach guarantees the reliability of the simulation and allows a strong reduction of the time needed to develop the digital prototype. We have extended this approach also to the MR, in order to overcome the lack of tactile feedback typical of the VR approaches [18]. The proposed MR environment is designed to manage the occlusion between real and virtual objects and to interpret the user's gestures while he/she is interacting with the elements of the product interface. The use of this MR environment was validated through some user tests addressed to establish the effectiveness of the proposed approach in the usability assessment of industrial products [19]. Taking into account the feedback collected from the users who have taken the tests, we improved the set-up with a custom-made interactive thimble that ensures a better comfort for the user and avoids the use of expensive interaction devices like data-gloves or tracking systems.

\section{The MR environment}

The hardware set-up used in this study (Fig. 1) is very easy to realise and it can be implemented with a very low budget. A video see-through HMD is used for augmented visualisation. The HMD is composed of an iWear VR920 and a CamAr, both from Vuzix. The CamAr camera has a resolution of $800 \times 600$ pixels at $30 \mathrm{~Hz}$. The camera is used both for the video see-through function and for marker-based tracking through the ARToolkitPlus library.

Instead of the glove previously used in [18, 19], we have currently adopted a thimble equipped with a micro-switch and an infrared LED. The user wears the thimble on his/her index finger tip. The micro-switch provides a force-feedback reaction when the user is pressing a virtual button, so he/she can feel the sensation of touching a real button on the physical prototype. The micro-switch activates the LED, so the pressing action is captured by the infrared camera. Compared to the other solutions presented in the state of the art, this MR environment does not require any pens [7] or tracking devices [4] to recognise the user's actions while he/she is interacting with the digital product. Compared to our previous works [18, 19], it does not require the glove, but just a more comfortable thimble.

The rendering pipeline (Fig. 2) designed for the application is structured as follows:

1. An image of the scene is acquired through the camera mounted on the HMD;

2. The camera image is displayed on the background of the frame buffer;

3. The tracking algorithm identifies the markers present in the scene and updates the position of the virtual prototype;

4. The virtual prototype is rendered in its proper position;

5. The hand-region is detected in the current frame and stored as a single bit hand-mask;

6. The index finger tip is tracked by applying a blob detection procedure on the hand-mask;

7. The position of the fingertip is used to check if the user is pressing a button of the product interface. Any trigger generated by the button pressing is sent to Simulink;

8. Simulink processes the triggers and updates the state variables;

9. Simulink sends the new values of the state variables to Virtools;

10. The $3 \mathrm{D}$ model of the digital prototype is updated considering the new values of the state variables;

11. The digital prototype is rendered on the frame buffer;

12. The user's hand is drawn on the frame buffer considering the hand-mask stored at the step 5;

13. The final image is then displayed on the HMD.

The correct visualisation of the user's hand in the MR environment requires the recognition of the skin that has to be separated from the background as shown in Fig. 3. 
Fig. 1 The MR set-up used in product simulation

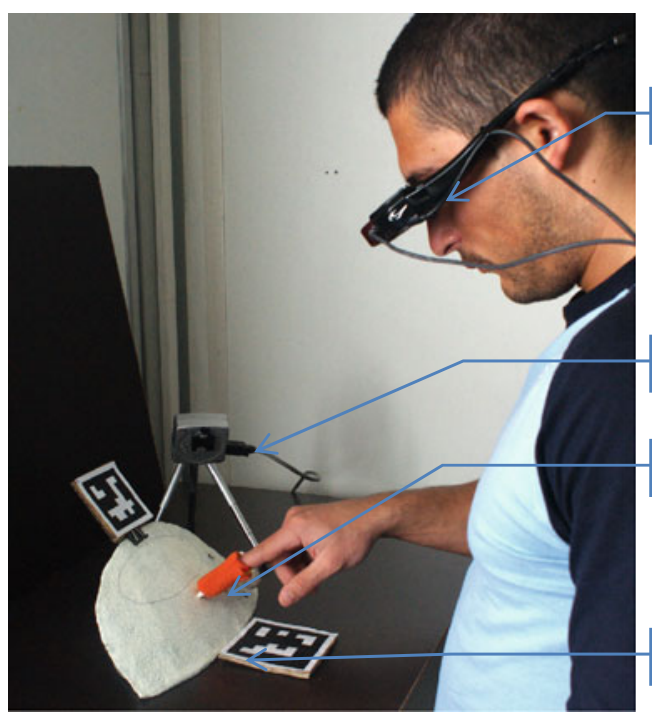

iWear VR920 with CamAr

Firewire infrared camera for finger tracking

Thimble with micro-switch and infrared led
Fig. 2 The rendering pipeline of the MR environment
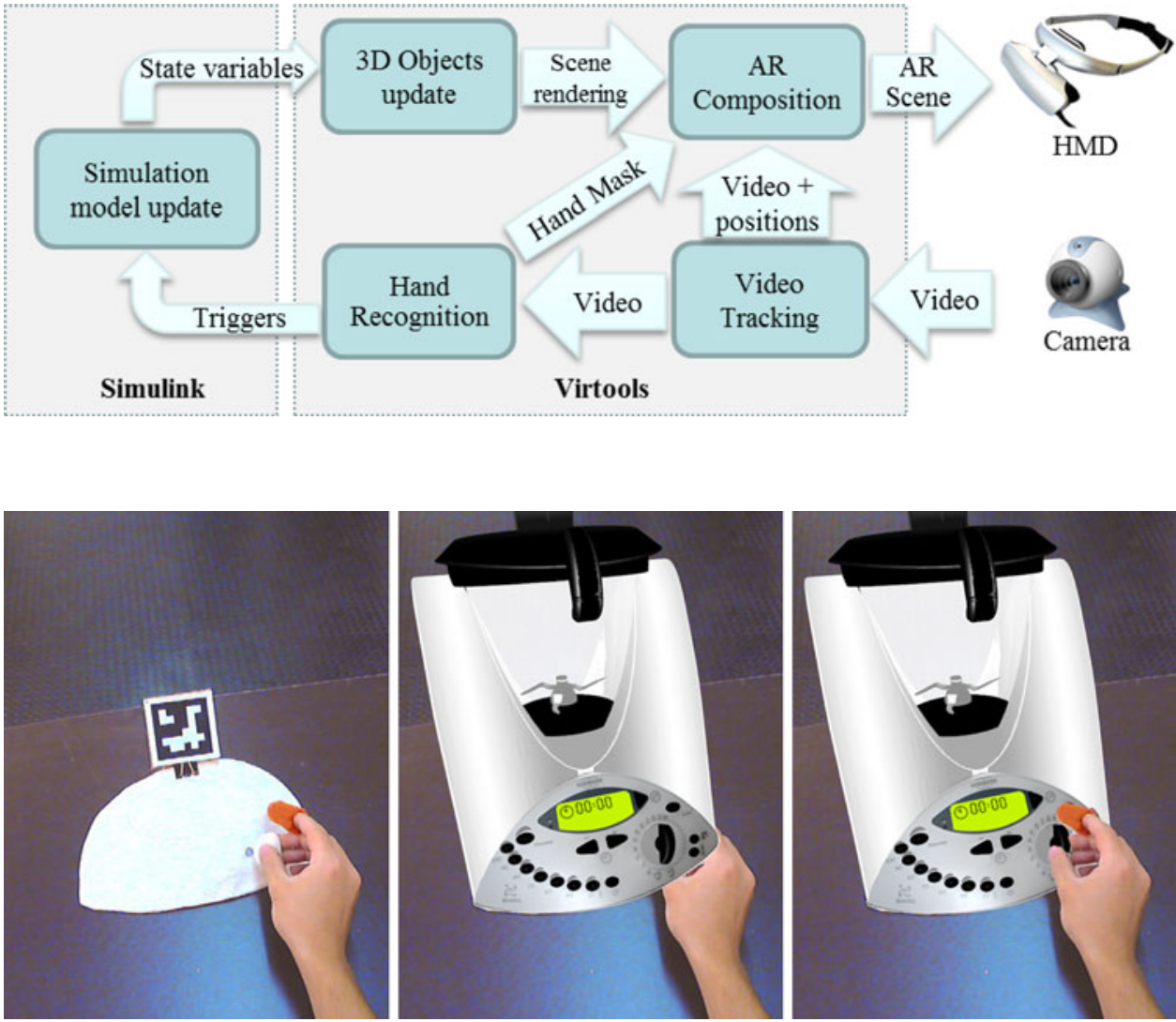

Fig. 3 The Mixed prototype with occluded (centre) and correct (right) hand visualisation
Dozens of previous researches have approached this issue by analysing the camera images in the different colour spaces [20-22]. Several techniques are proposed in literature and there are some comparisons that highlight the advantages and disadvantages of each algorithm. We have adopted the hue, saturation, intensity (HSI) colour space to overcome the illumination problems like high intensity at white lights, ambient lights or surface orientation relative to the light source. We have improved the technique previously adopted in $[18,19]$ by implementing a multi-pass shader that, after the image segmentation, tries to reduce the false positive and the false negative pixels. The first pass of the shader performs the segmentation in the HSI colour space; the second one recognises and deletes isolated areas, in order to eliminate the false positive pixels; the third and fourth passes fill the 


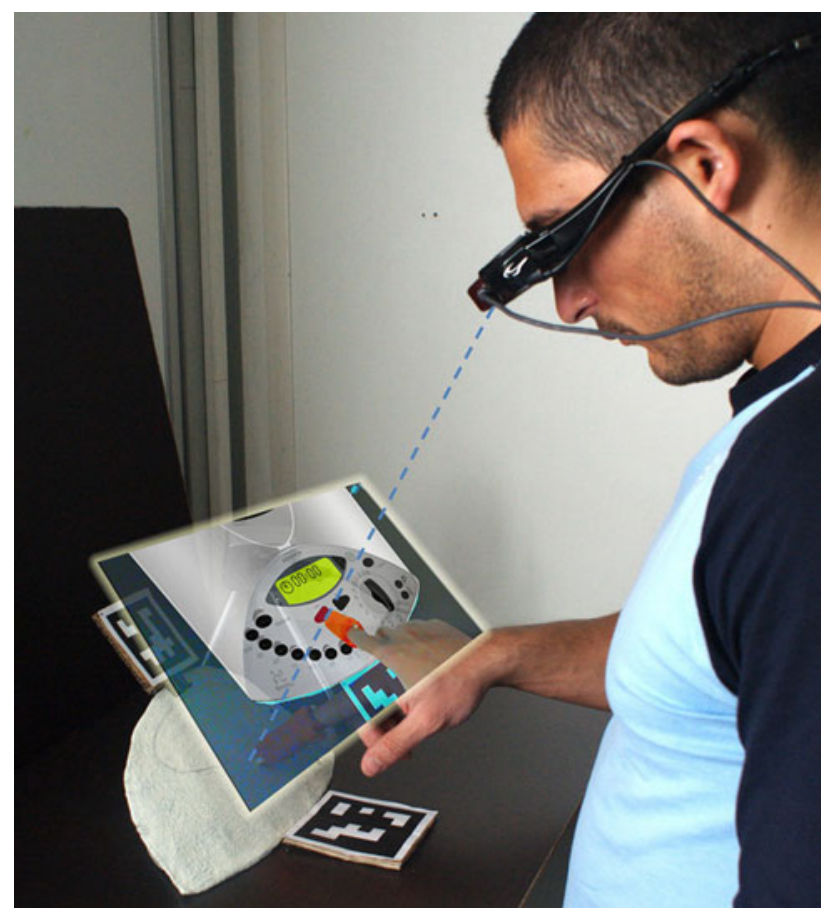

Fig. 4 Illustration of the ray casting technique used to check whether the user is pressing the HMI buttons

holes inside the segmented area, in order to eliminate the false negative pixels. The shader recognises multiple colours, so it is able to segment both the skin and the thimble on the same pass.

The adopted tracking strategy determines the position of the user's index finger tip in the screen space coordinate system through a blob detection algorithm. The $2 \mathrm{D}$ position of the index finger tip is used to determine where the user is pointing, through a classical ray casting method. When the user attempts to push a button of the virtual product, his/her index finger impacts on the physical prototype, activating the micro-switch mounted on the tip. The activation of the micro-switch triggers the ray casting to detect whether the user is activating any button of the product (Fig. 4).

The approach described until now manages the user's interaction with the buttons of the HMI. To apply the same methodology to more complex interfaces (containing other widgets like knobs, sliders, or leverages), we follow the approach described in [23] that proposes to equip the rapid prototype with some interactive elements. For this reason, we placed a knob on the surface of the physical prototype (as depicted in Fig. 3; left) connected to an encoder that determines any rotation of the knob itself. This choice is motivated by the fact that the rotation of a virtual knob is a complex task for the user. The presence of the tangible knob strongly simplifies the interaction with the prototype.

\section{Interactive digital prototypes}

In this work, we define an "interactive digital prototype" as a digital model of the product that interactively responds to the action performed by the user through the product interface. This section describes an original approach for the creation of interactive digital prototypes based on the coupling of the visualisation software with a simulation environment. This idea is mainly justified by the fact that, in many cases, the behaviour model of the product is already available since the first steps of the product development process. In fact, control engineers usually realise a behaviour model of the product using a computer aided control engineering (CACE) tool. This model is used not only to schematise the product behaviour, but also to analyse how it works with a proper simulator.

\subsection{Actors of interactive digital prototyping}

Figure 5 schematises the proposed approach and the role of the four actors involved in the process, including the above-mentioned control engineer. The designer defines the geometries of the product and, in particular, the external shape, that is essential from the aesthetical point of view, and the appearance of the interface.

The VR operator uses an authoring tool to prepare the virtual environment specifying which 'input elements' of the interface (button, knob, etc.) may be controlled by the user and which 'output elements' (LED, LCD, etc.) may change their status during the simulation. On the other side, the control engineer defines the behaviour of the product using CACE tools. Then, he/she connects the state transition of the model with the user-generated events and the state variables with the 'output elements' of the user interface (UI) defined in the virtual environment. The link between the two environments requires a specific tool that will be described in the following section. Adopting this approach, the behaviour of the virtual prototype is exactly the same as the one the control engineer has defined in the CACE models used for his/her design and analysis activities. The three actors that we have identified on the left part of Fig. 5 have to be intended as "roles played in the design process". In some cases, generally in small enterprises, some of these roles may be played by the same person. For example, the virtual environment may be prepared by the designer or by the control engineer. Extending this reasoning, we may assume that all the three roles may be played by only one actor with all the competencies.

We suppose that the end user is one of the actors of the process because, as clearly evidenced in literature [2-7], VR and MR tools are particularly effective when the user involvement in the testing phase is needed. In other terms, our approach allows the user to test what engineers have designed by a direct interaction with the digital prototype. 
Fig. 5 The schema of the approach proposed for mixed prototyping

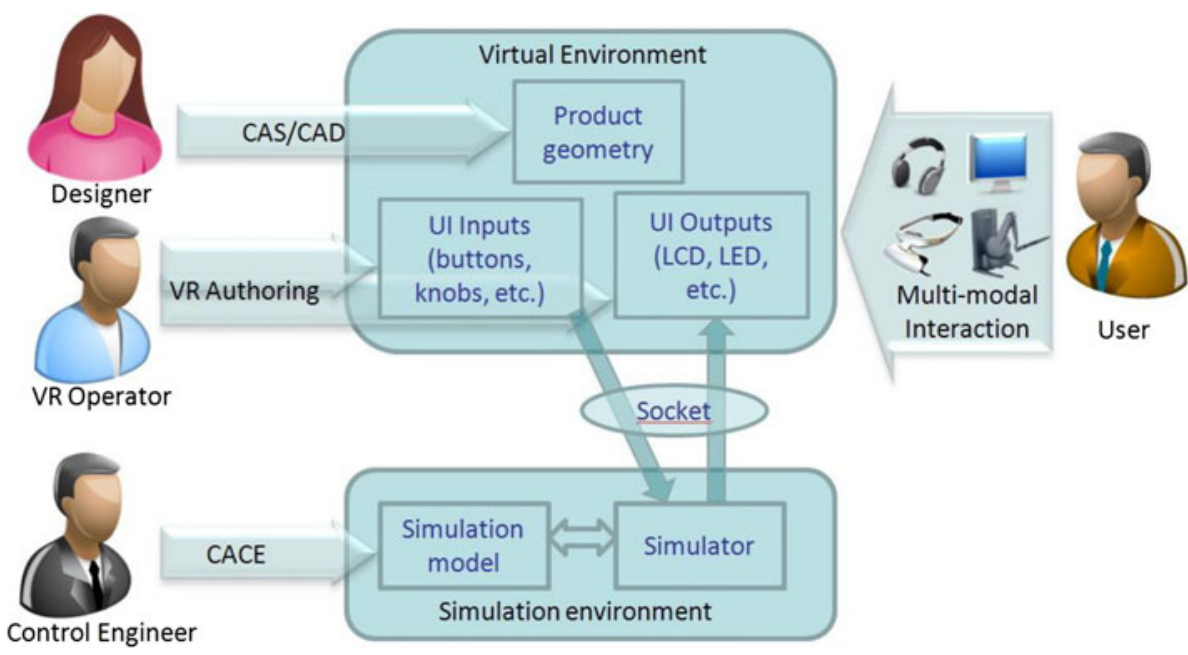

\subsection{Software modules}

In this paper, we present a case study to demonstrate how it is possible to realise an interactive digital prototype connecting a simulation software with a visualisation tool for VR/MR. In particular, we have chosen Matlab/Simulink to model and simulate the product behaviour. This environment is almost a standard for the general purpose simulation, thanks to its diffusion and versatility. Furthermore, a lot of optional packages (called toolboxes) are present. These packages provide further sets of high level operations for a specific task. They make it easier to implement the real-time simulation engine needed to fully reproduce each kind of behaviour. For the visualisation, we have employed Virtools Dev because it is one of the most powerful authoring environments for interactive 3D graphics and VR. Moreover, Virtools Dev can be extended through its own $\mathrm{C}++$ application program interface (API). All implementation details about MR authoring task in Virtools, as well as the CACE simulation implementation in Simulink, are given in Sect. 5 through the description of the test case.

The choice of Matlab/Simulink as a simulation environment is mainly motivated by the great flexibility of this product that can be easily extended with optional packages or custom-made add-ons. This makes it possible to include in the simulation different modules that may cover several engineering domains (electrics, electronics, dynamics, thermal, etc.). In the easiest case, the engineer may simulate only the logics of the user interface through a finite state machine (FSM) or similar modelling languages. In comparison with the other solutions present in literature that use custom tools [14] or specific modelling languages [16], the adoption of Simulink may allow the engineers to realise complex models of the product behaviour, specifying also the functional aspects of the product. This means that most of the internal components (e.g. electric motor, mechanical parts, hydraulic circuits, etc.) may be simulated using specific toolboxes available in Simulink. In the test case (see Sect. 5.1), an electric and an electronic model were also included, in order to simulate the physical behaviour of the electric motor and the relative control circuit.

Simulink was also chosen because it was able to fulfil all the simulation needs related to the test case described in Sect. 5, but, in general, the choice of the simulation environment should be evaluated taking into account the complexity of the product interface and the specific functionalities that the designers want to simulate. In the case of information appliances, e.g., it would be useful to adopt a simulation tool like Altia Design, or d.tools [24] that make the design and simulation of complex product interfaces easier.

\subsection{The link between simulation and MR}

The data exchange between Simulink and Virtools has been realised developing some custom add-ons for both the environments. These add-ons are based on a software library called SimLib that was previously developed for dynamic simulation in VR [9]. This library provides an inter-process communication (IPC) channel using TCP socket. The SimLib has few functions, implementing the code for TCP/IP communication and synchronisation. Therefore, the developer must not take care of sockets and threads.

To integrate the SimLib inside Simulink, we have developed two custom S-Functions, responsible for the communication between Simulink and Virtools through the IPC channel. One S-Function receives the events generated by the user that interacts with the virtual product and switches different simulation parameters according to these 


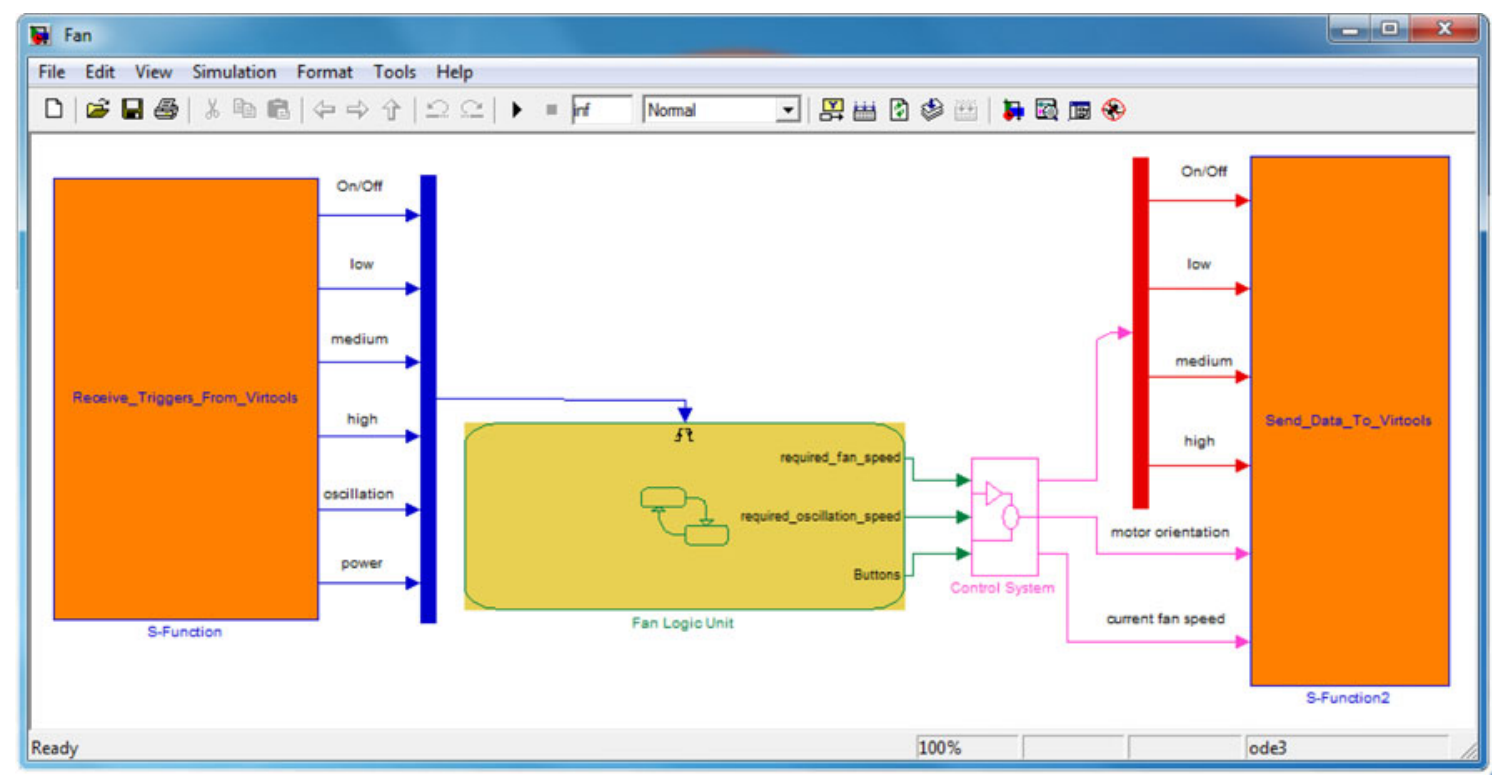

Fig. 6 A simple use of the SimLib in a Simulink model

events. The other S-Function sends the data that describe the state of the product and each change that occurs during the simulation. The data sending is based on an asynchronous channel: the S-Function sends data to Virtools, without stopping the simulation. To obtain a consistent visualisation, in fact, it is necessary to send all data at each time-step simultaneously.

Figure 6 shows an example of these S-Functions used in an electric fan. The S-Function on the left receives six triggers generated by the button press events. The triggers are processed by a FSM and a control system module. The updated state variables are sent to Virtools thanks to the S-Function on the right.

On the Virtools side, the IPC has been implemented through two user defined building blocks (BB). A BB is like a black box with some parameters in input and some in output. By connecting several BBs, it is possible to create an interactive application in Virtools. The two BBs we have implemented to support the connection with Simulink are able to send and receive data through the IPC channel. The first BB sets the 3D model parameters in agreement with the data coming from Simulink. The second BB sends to Simulink the messages generated by the user interaction in the mixed environment (e.g. a button pressed, a knob rotated, etc.).

\subsection{Creation of the digital prototype}

We assume that the Simulink model can be realised following a top-down process where the product behaviour is progressively refined until each component of the product has been defined. For instance, the model we have realised for the test case, as described in Sect. 5, can be considered as the result of the first stage of the design process. In the subsequent steps of the process, the model could be refined by transforming the behaviour model (made of functions, formulas and equations) in a schema where each block represents a physical component (e.g. electric motor, actuators, processors, signal converters, etc.).

The geometric model of the product is usually defined following a bottom-up approach. Using a CAD system, the engineers model each single component or import them from previous projects or from the suppliers' catalogues. But, in order to realise a virtual test with the product interface, only the external shape of the product is needed. All the components inside the object that are not visible to the user can be omitted in the virtual prototype. This allows engineers to start the test with the users as soon as the 'skin' of the product has been defined by the designers. When the shapes are ready, these are imported into the virtual environment and the properties of the interface elements are connected to the state variables of the simulation model. Then, the events generated by the user are addressed to the simulator that considers them as triggers for the state transitions. In most cases, the connection between the simulator and the visualisation software does not change during the process because the exchanged data remain the same also when the behaviour model is updated and refined.

In the general case, at the end of the design process, several different models are defined for each engineering domain involved in the product. For example, in our test case we could have the Simulink model that simulates the electric and electronic behaviour and the virtual prototype 
with all the CAD geometries of each part assembled in a unique digital mock-up. The latter can also be used to realise multi-body and/or structural analyses using CAE software. Different models can also be used together to realise a mechatronic co-simulation coupling different simulators or using an integrated simulation environment.

\section{The test case}

The test case we have analysed to validate our virtual prototyping approach is a hot mixer (Fig. 7). This product combines cooking and mixing functions in the same device and is used to prepare a wide variety of foods. It is a rather complex appliance with several functions like weighing, blending, grinding, kneading, steaming and cooking.

The product contains a classic interface consisting of a knob, which allows the user to set the speed of the motor, eight buttons to set the temperature, a couple of buttons to set the timer, the stand-by button, and other three buttons for special functions: turbo, weighing and counter-clockwise rotation. It has also an LCD that displays the state of the mixer (timer, weight, functions activated) and a set of eight LEDs associated with the temperature buttons that show the current and the desired temperature.

\subsection{Design and simulation of the product behaviour}

The realisation of an interactive digital prototype implies the formalisation of the product interface behaviour in a

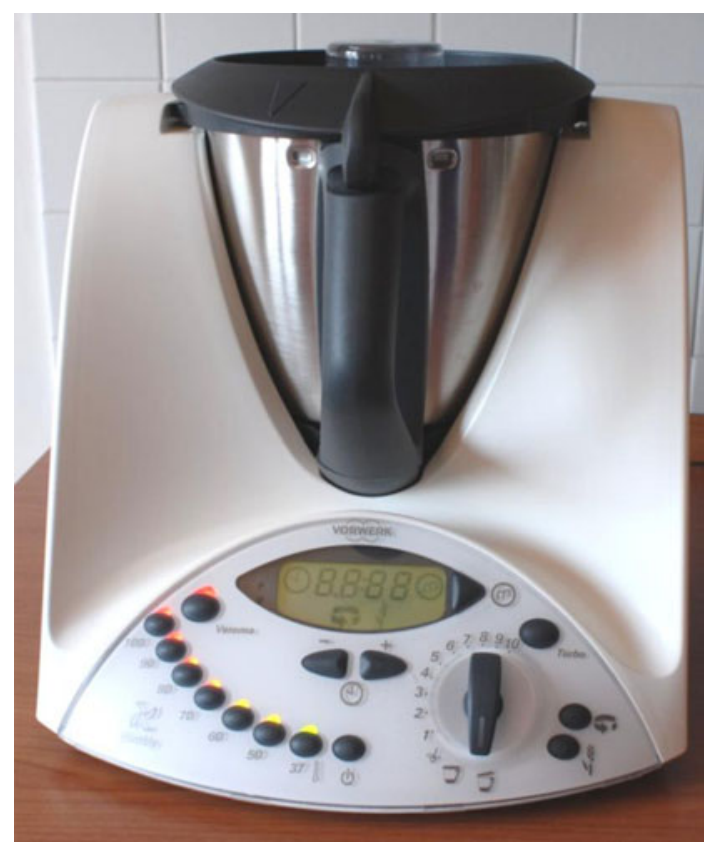

Fig. 7 The hot mixer used as test case to validate the virtual prototyping approach proposed in this paper simulator able to process the user's inputs so as to determine how the status of the product changes. Therefore, the behaviour of the product interface could be schematised using a FSM model.

The behaviour model of the product was realised in Simulink using the StateFlow toolbox that supports the creation of FSM, combining hierarchical state machine diagrams of the kind introduced by statecharts [15] with traditional flowchart diagrams.

Figure 8 shows the complete Simulink model of the product. The FSM, labelled as "logic unit", is located in the left part of the picture. The FSM has two kinds of input: the first one, on its left, is related to the knob that controls the speed of the motor. It consists in two arrays that, for each angle of the knob rotation, specify the speed of the motor. The other input, on the upper part of the FSM block, is a bus that transmits all the data received from Virtools through the socket connection managed by the orange block.

The user actions are processed by Virtools, which collects all the user-generated events (button pressed, knob rotated, cover opened/closed) and sends them to Simulink. It receives all these data through the "receive from Virtools" block. Also the clock signal is transmitted because it is used to synchronise the two applications. The "logic unit" continuously update the state of the simulated product, based on the received inputs and the current state. Then, the current configuration is iteratively sent back to Virtools (see the "send to Virtools" block in Fig. 8), in order to update the model and simulate the behaviour of the interactive virtual prototype.

The FSM has seven outputs. The first value, $O R$, is the current angular orientation of the knob. The following four values, $m d, m u, s d, s u$, are the four digits on the LCD. $V E L \_$desired is the desired motor speed set by the user with the knob, led is a vector that contains the state (on/off) of each temperature LED. The desired motor speed is processed by a block that defines the physical behaviour of the electric motor and the relative control circuit using the SimScape and the SimElectronics toolboxes.

\subsection{Creation of the MR environment}

The design of the behaviour model of the product, described in the previous section, usually represents one of the first steps in the design process of the control system for an electric appliance. With our approach, even this preliminary model can be used to evaluate the functions and the usability of the product interface. A digital mock-up of the product is needed to test, with the user, the previously described behaviour model. As previously mentioned, the geometric model of the product has been reconstructed by measuring the real product. This geometry, completed with 


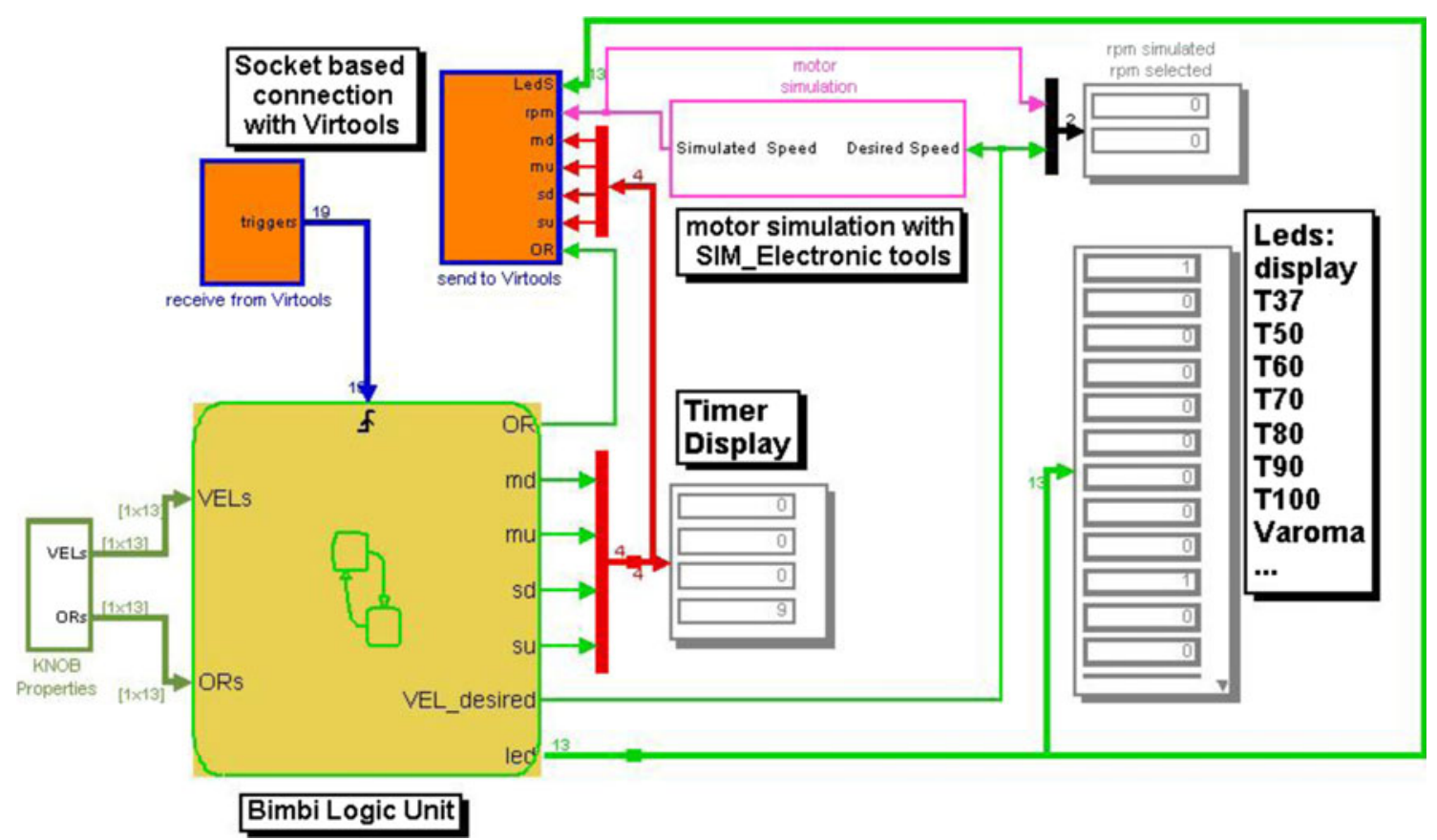

Fig. 8 Simulink model of the product

the materials and the textures, has been imported in Virtools. After that, the connection with the Simulink model has to be created. This activity consists of the following tasks:

1. To define the state variables that have to be connected to the Simulink model. The present test case has six state variables that are the outputs of the FSM described in the previous section.

2. To create a socket receive $\mathrm{BB}$ able to process the data stream sent by the Simulink model.

3. To connect each output of the socket receive $\mathrm{BB}$ to its relative state variable.

4. To identify all the possible user-generated events and catch them through a switch on message BB. This BB sends a message to the Simulink model each time that the user generates an event.

The user-generated events received by the switch on message BB may be button pressed and knob rotated.

The display is simulated by defining, on the virtual model of the product, a series of rectangular patches on which different textures are mapped; such textures are changed at run-time according to the values of the different variables that define the state of the product. The display is divided into nine parts: four are for the digits used to display time and temperature, one for the colon between the digits, four are used to show the status of some functions (timer, weighing, kneading, counter-clockwise rotation) (Fig. 9). The textures representing the ten digits are stored in a vector and assigned to the four slots according to the messages received by the Simulink connection.

Figure 10 is a screenshot taken while the simulation is running. In the upper part of the image, the Virtools window shows the interface with the timer at 01:21; the temperature is currently at $37^{\circ} \mathrm{C}$ (yellow LED) and it has to reach $70{ }^{\circ} \mathrm{C}$ (orange flashing LED); the speed is set to 3 . In the lower part of the image, the simulation running in Simulink is illustrated. The state variables present the same values of temperature, time and speed seen on the Virtools model.

\subsection{Considerations about the test case}

The implementation of the test case allowed us to evaluate the effectiveness of the proposed approach and, in particular, the advantages related to the creation of a direct link

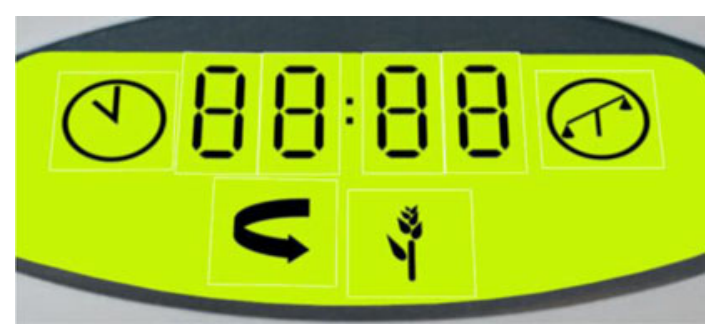

Fig. 9 The display of the product is made of nine rectangular patches with different textures that are changed at run-time by the simulator 


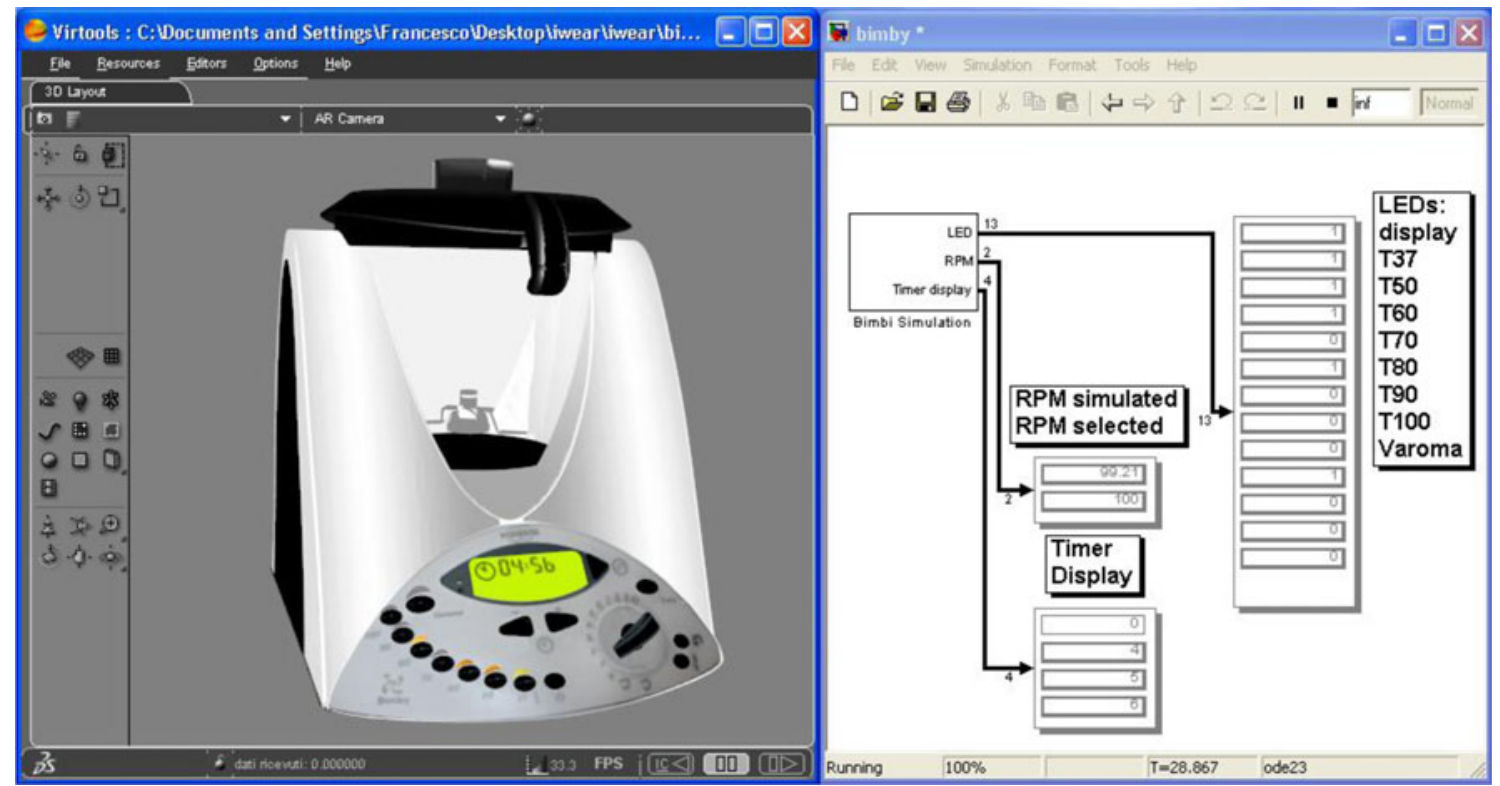

Fig. 10 The simulation of the hot mixer running simultaneously in Virtools (left side of the image) and Simulink (right side)

between the simulation and the visualisation environment. To carry out this evaluation, we have developed a virtual prototype of the same product analysed in the test case, without using the connection between Virtools and Simulink. In other words, we have simulated what happens in a virtual prototyping practice conducted using two separate environments for control engineering and VR. In this situation, the VR operator has to read and understand the Simulink model developed by the control engineer and has to replicate the same behaviour in Virtools using a combination of behavioural graphs and pieces of code written in Virtools scripting language (VSL). However, an exact replication of the behaviour is practically impossible because the Simulink model includes several complex functions of the Simulink toolboxes used to simulate the FSM and the sub-model of the motor control circuit. These functions are necessarily approximated by the VR operator because a precise re-implementation is practically impossible. For this reason, the VR operator had to perform several tests on the behavioural model implemented in Virtools to check if it works exactly as the original one.

Moreover, it is apparent that, if the control engineer modifies the Simulink model, he/she has to track each change to explain to the VR operator how to update the behavioural model implemented in Virtools. This means that the time that may be saved using the proposed approach is proportional to the number of modifications done on the behavioural model during the iterations of the design cycle.

The last consideration concerns the knowledge sharing issue. In our study, the VR operator had a good knowledge of Simulink, so he was able to directly interpret the behavioural model. But, in general, the control engineer and the VR operator shall adopt a formalism to schematise and share the behavioural model. With our approach, the communication between the control engineer and the VR operator is strongly reduced because they have to share only the data concerning the triggers and the state variables that have to be exchanged between the simulation and the VR environment. This is an important simplification, which may allow saving a lot of time.

To evaluate other possible alternatives to the proposed approach, we have considered the 3D Animation toolbox available in the Matlab Environment. With this toolbox, in fact, it is possible to control a VRML model visualised in a 3D window. In particular, it allows to link the Matlab variables to the position, orientation and scale of the VRML nodes, thus obtaining an animation of the product synchronised with the simulation. Obviously, this approach presents several limitations because the 3D Animation toolbox does not support AR visualisation and other useful computer graphics techniques like ray casting and shaders that are needed to implement the interaction technique proposed in Sect. 3.

Summarising the results of this analysis, the case study proves that, with the proposed approach, it is possible to obtain a reliable simulation of the product behaviour without any recoding or approximation in the logics definition; it is possible to clearly separate the tasks assigned to the control engineer from the ones assigned to the VR operator; it is possible to quickly modify the behaviour directly within the simulation environment; it is possible to take advantages of all the features available in a software like Virtools that, in general, is more powerful of the 3D 
Animation toolbox included in the simulation environments. All these possibilities also contribute to achieve a reduction of the time needed to develop a virtual prototype.

On the other side, the main limitation of the proposed approach is that Matlab has to be able to perform an interactive simulation with an update rate that should be at least $25 \mathrm{~Hz}$. This requirement is needed to give a realistic feedback to the user's actions, but it limits the possibility to use complex behaviour models. For this reason, it should be useful to evaluate the possibility to extend this approach to include other simulation environments that may give better computational performances.

\subsection{Test with the users}

In a previous work [19], we analysed the influence of the MR environment in the assessment of the usability of virtual products: we verified that the simulation of the product in MR did not invalidate the result of the usability evaluation. The study compared the usability tests (performed on a microwave oven) done in real and VR environment with the one done in MR. The comparison focused on the benefits (in terms of efficacy, efficiency and satisfaction of use) and the limitations related to the two setups. The most valuable result of this study was that, regardless of the type of interaction taken into consideration (whether real, virtual or augmented), the same difficulties of functional understanding of the product were pointed out by all the users.

Since the effectiveness of the proposed approach has already been verified in [19], in this paper we address our evaluations to the assessment of the acceptability among end users of the MR set-up through a satisfaction questionnaire.

To carry out the user test, we chose a sample of 10 mechanical engineering students aged between 21 and 24 . The sample size is large enough to reveal most of the usability problems as stated in different works about usability evaluation [25-27]. All users presented homogeneous features especially concerning the knowledge of the product we were testing. In particular, before the test, we asserted that they did not know the product at all, so we were also able to verify whether they were able to focus on the task of understanding the product without being influenced by the use of the MR system.

The experiment was carried out in three main phases: an analytical phase during which we organised the protocols of the test; an operational phase during which the users performed the test with the virtual product; an assessment phase during which we analysed all the collected information.

During the analytical phase, we prepared two questionnaires: the first one aimed to identify the users' profiles, the second one to evaluate the users' satisfaction after the test of the MR system. Moreover, in this phase, we defined the tasks to be undertaken by users during the test.

During the operational phase, each user carried out four activities: filling in the user-profile questionnaire; exploring the MR environment; interacting with the mixed prototype; filling in the questionnaire on the degree of satisfaction.

During the interaction with the product, we asked the user to execute four tasks presented as realistic scenarios. After the interaction phase, we asked the users to fill in the satisfaction questionnaire, made up of four questions. The collected data allowed us:

- To evaluate the users' overall degree of satisfaction and their well-being or sense of unease perceived after having used the MR devices (in particular HMD and thimble);

- To identify the problems related to specific aspects of the interaction.

In the past 30 years, the studies about usability have introduced a large variety of potential usability measurements that can been categorised in different classes. However, in our experiment we have adopted as metrics those suggested by general agreement from the standards ANSI 2001 [28] and ISO 9241 [29]. In particular, we have adopted the degree of satisfaction using a satisfaction questionnaire based on psychometric methods, as defined in [30].

The main results related to questionnaire are shown in Fig. 11. The first two questions show that the easiness of use and the agreeableness are good. The predominance of "low" in the third question indicates that the users are not frustrated by the interaction with the system. The only aspect that does not completely satisfy the users is the perception of the texture. This problem is mainly due to the low resolution of the HMD. It is reasonable to assume that a better HMD and a better camera could improve the overall quality of the visualisation.

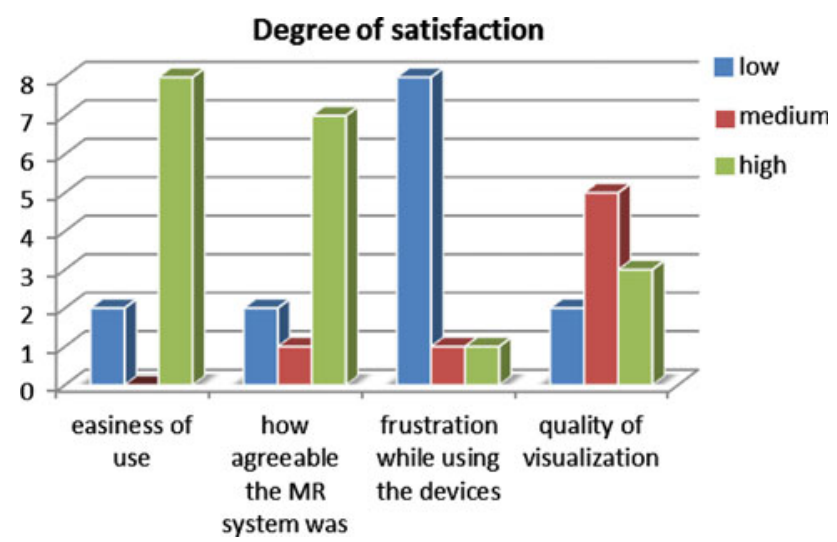

Fig. 11 Degree of satisfaction of users related to the use of MR devices 


\section{Conclusions}

In this paper, we have presented an innovative approach to virtual and mixed prototyping based on the idea that the CACE software, usually employed by the control, electrics or electronics engineers, can also be used to simulate the behaviour of the product HMI in VR/MR. This approach overcomes the classical problems related to the implementation of the code inside the VR/MR software to replicate the product behaviour. The implemented test case puts in evidence that the main advantages of our approach are

- The reliability of the HMI simulation in VR/MR is ensured by the direct use of the same solver and the same behaviour models that engineers use to design and manufacture the product.

- The time needed to implement an interactive digital prototype is reduced because there is no need of coding to model the HMI behaviour.

- Any modification done on the behaviour models in the simulation environment is immediately testable in VR/MR.

The paper also presents a MR environment aimed to overcome two different issues typical of the product behaviour simulation in MR: the occlusion between the user's hands and the virtual objects and the interaction between the user and the product HMI. Given these challenges, we developed an interaction technique that relies on the real-time processing of the HMD video stream. Our test confirmed that the proposed technique is able to handle the occlusion issue and to recognise the user-generated event.

The implementation of the test case shows that the proposed approach may be effective for the design of electric appliances where the user interface is made of electromechanical elements like buttons, knobs, LEDs and displays. Further development will focus on the implementation of other test cases by considering products with a more complex user interface characterised, for example, by the presence of many small buttons (e.g. television remote control) or by the presence of elements like touch screens. In these cases, the easiness of the interaction could suffer from the low precision of the finger tracking technique. Also the interaction through more fingers and/or both hands should be considered as a possible improvement. Moreover, the integration with other simulation environments (e.g. OpenModelica, Dymola, Comsol, SimXpert, etc.) should be implemented to have the possibility to adopt the solver that best fits the needs of the specific case of product simulation.

\section{References}

1. Jimeno A, Puerta A (2007) State of the art of the virtual reality applied to design and manufacturing processes. Int J Adv Manuf Tech 33-9(10):866-874

2. Bruno F, Muzzupappa M (2010) Product interface design: a participatory approach based on virtual reality. Int J Hum Comput Stud 68-5:254-269

3. Barbieri L, Bruno F, Caruso F, Muzzupappa M (2008) Innovative integration techniques between Virtual Reality systems and CAx tools. Int J Adv Manuf Tech 38-11(12):1085-1097

4. Aoyama H, Kimishima Y (2009) Mixed reality system for evaluating designability and operability of information appliances. Int J Interact Des Manuf 3-3:157-164

5. Bordegoni M, Cugini U, Caruso G, Polistena S (2009) Mixed prototyping for product assessment: a reference framework. Int J Interact Des Manuf 3-3:177-187

6. Park H, Son JS, Lee KH (2008) Design evaluation of digital consumer products using virtual reality-based functional behaviour simulation. J Eng Des 19-4:359-375

7. Park H, Moon HC, Lee JY (2009) Tangible augmented prototyping of digital handheld products. Comput Ind 60-2:114-125

8. Bruno F, Caruso F, Muzzupappa M, Stork A (2007) An experimental environment for the runtime communication among different solvers and visualisation modules. Proceedings of 19th European Modeling and Simulation Symposium, Genova, Italy

9. Bruno F, Caruso F, Li K, Milite A, Muzzupappa M (2009) Dynamic simulation of virtual prototypes in immersive environment. Int J Adv Manuf Tech 43-5(6):620-630

10. Fiorentino M, de Amicis R, Monno G, Stork A (2002) Spacedesign: A mixed reality workspace for aesthetic industrial design. Proceedings of the International Symposium on Mixed and Augmented Reality (ISMAR02), Darmstadt, Germany

11. Verlinden J, Horvath I (2006) Framework for testing and validating interactive augmented prototyping as a design means in industrial practice. Proceedings of Virtual Concept, Playa Del Carmen, Mexico

12. Verlinden J, Horvath I (2007) A critical systems position on augmented prototyping systems for industrial design. Proceedings of the ASME-CIE 2007, DETC2007-35642, International Design Engineering Technical Conferences, Las Vegas, USA

13. Verlinden J, Horvath I (2009) Analyzing opportunities for using interactive augmented prototyping in design practice. Artif Intell Eng Des Anal Manuf 23:289-303

14. Kanai S, Higuchi T (2008) Applying user interface-operable 3D digital prototypes to human-centered design of information appliances. Proceedings of the ASME 2008 International Design Engineering Technical Conferences \& Computers and Information in Engineering Conference IDETC/CIE 2008, New York, USA

15. Harel D (1987) Statecharts: a visual formalism for complex system. Sci Comput Program 8:231-274

16. Kanai S, Higuchi T, Kikuta Y (2009) 3D digital prototyping and usability enhancement of information appliances based on UsiXML. Int J Interact Des Manuf 3-3:201-222

17. Bruno F, Angilica A, Cosco F, Muzzupappa M (2010) Functional behaviour simulation of industrial products in virtual reality. Proceedings of the 8th International Symposium on Tools and Methods of Competitive Engineering, TMCE 2010, 2, pp 763-774

18. Bruno F, Angilica A, Cosco F I, Muzzupappa M (2010) Product behaviour simulation in mixed reality. Proceedings of ASME World Conference on Innovative Virtual Reality (WINVR2010), Ames, Iowa, USA 
19. Bruno F, Cosco F, Angilica A, Muzzupappa M (2010) Mixed prototyping for products usability evaluation. Proceedings of the ASME 2010 International Design Engineering Technical Conferences \& Computers and Information in Engineering Conference (IDETC/CIE 2010), Montreal, Quebec, Canada

20. Vezhnevets V, Sazonov V, Andreeva A (2003) A survey on pixel-based skin color detection techniques. Proceedings of Graphicon 2003, Moscow, Russia

21. Kakumanu P, Makrogiannis S, Bourbakis N (2007) A survey of skin-color modeling and detection methods. Pattern Recogn 40-3:1106-1122

22. Phung SL, Bouzerdoum A, Chai D (2005) Skin segmentation using color pixel classification: analysis and comparison. IEEE Trans Pattern Anal Mach Intell 27-1:148-154

23. Bordegoni M, Caruso G, Ferrise F (2008) Mixed-Reality Environment based on Haptic Control System for a tractor cabin design review. Proceedings of CIRP Design Conference, Enschede, April 7-9

24. Hartmann B, Klemmer SR, Bernstein M, Abdulla L, Burr B, Robinson-Mosher A, Gee J (2006) Reflective physical prototyping through integrated design, test, and analysis. Proceedings of the Annual ACM Symposium on User Interface Software and Technology-UIST'06, Montreux, Switzerland

25. Landauer TK, Nielsen J (1993) A mathematical model of the finding of usability problems. In: Proceedings of ACM INTERCHI'93 Conference, Amsterdam, 24-29 April, 206-213

26. Virzi RA (1992) Refining the test phase of usability evaluation: how many subjects is enough? Hum Factors 34:457-468

27. Nielsen J (2000) Why you only need to test with 5 users: Alertbox. http://www.useit.com/alertbox/20000319.html

28. ANSI (2001) Common industry format for usability test reports (ANSI-NCITS 354-2001). American National Standards Institute, Washington, DC

29. ISO 9241 (1998) Ergonomic requirements for office work with visual display terminals (VDTs)-Part 11: guidance on usability (ISO 9241-11:1998(E)). Geneva, Switzerland

30. Lewis JR (2006) Usability testing. In: Salvendy G (ed) Handbook of human factors and ergonomics. John Wiley, New York, NY, pp 1275-1316 